\title{
Visualization and Interaction Research of Digital 3D Heart Model
}

\author{
Yang $\mathrm{Xu}^{1, \mathrm{a}}$ \\ ${ }^{1}$ School of Software, Beijing University of Technology, Beijing 100124, China; \\ aanglechi@126.com
}

Keywords: 3D heart model, interaction, visualization, internal structure, clinicopathologic analysis.

\begin{abstract}
The research of this paper can help medical researchers or ordinary people understanding the internal structure of heart and providing anatomic basis for clinicopathologic analysis of heart, heart anatomy teaching, surgical planning and visible simulation surgery of heart. Based on the heart anatomy data, use 3D Max to build the heart models and unfold the models in order to draw the textures. After that, combined with 3D model cutting algorithm, complete the three-dimensional interactive programming in Unity 3D, realize the interactive presentation of 3D heart model. The research has successfully established the detailed internal structure of 3D heart model and realized several functions such as moving, scale changing, rotation, independent show, transparent changing and real-time cross-section.
\end{abstract}

\section{Introduction}

Since the 1980s, people began to explore the application of computer technology in the field of medicine. In 1994, the combination of computer technology and medical science has been further promoted by the finish of Visible Human Project in United States. Since then, the 3D reconstruction of digital human body has became the research hotspot and formed the field of medical visualization gradually.

This paper researches the visualization and interaction of 3D heart in the field of medical visualization. With the development of digital media technology, people's understanding of heart isn't limited to 2D heart maps, entity heart model and heart specimens any more. Comparing with traditional technology, the 3D heart model generated by digital media technology can mobilize human senses such as vision, hearing preferably, it has realized a well interaction between human and computer.

\section{Materials and Methods}

Building 3D Heart Model. Based on the heart anatomy data, use 3D Max to build the heart models. The heart models are divided into four layers: epicardium layer, cardiovascular layer, atrioventricular muscle layer and cardiac chambers layer. The visible part of the outer layer of the human heart, called the epicardium, it is connected with adventitia and the connective tissue of myocardial layer, which contained many elastic fibers, blood vessels, lymphatic vessels, and nerves. Take out the epicardium of heart, we can see the myocardial layer and the cardiovascular layer around it. Take out the cardiovascular layer again, and dissect the myocardial layer, we can find all the structures in the cardiac chambers. After building the whole heart model, unfold them and draw the textures on them. The final results are as shown below. 


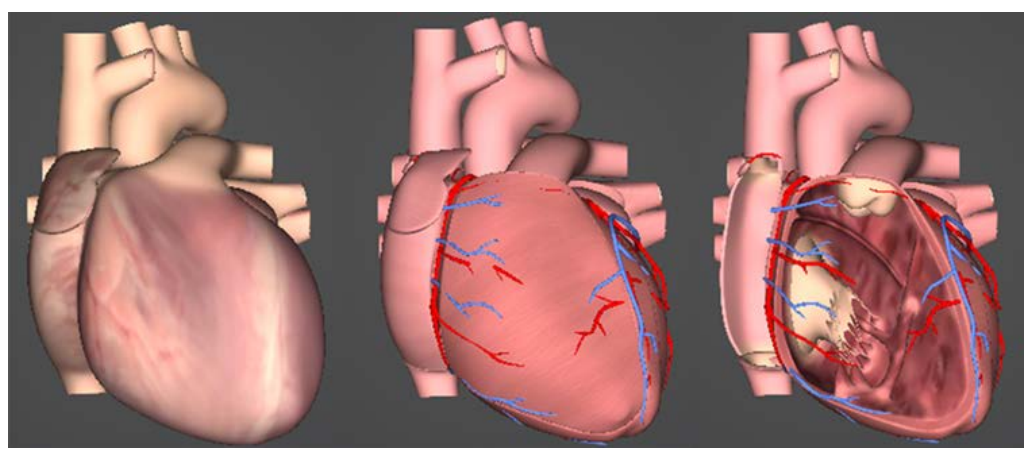

Fig. 1 Building 3D Heart Model

System structure design. In system development, visualization and interaction for digital 3D heart model is divided into seven parts to design and develop, namely heart model, interface design, lights, cameras, animations, text data and C\# script. The heart models consist of 20 sub model, the interface design including menu bar, toolbar, background and note cards, and the animation section contains three Animation videos. With lighting effect, 3D models and the interface has formed a visible part of the front through the cameras' range control, the complex interaction and logical relation between user, interface and the model are realized by the C\# script language. The structure design is show as below.

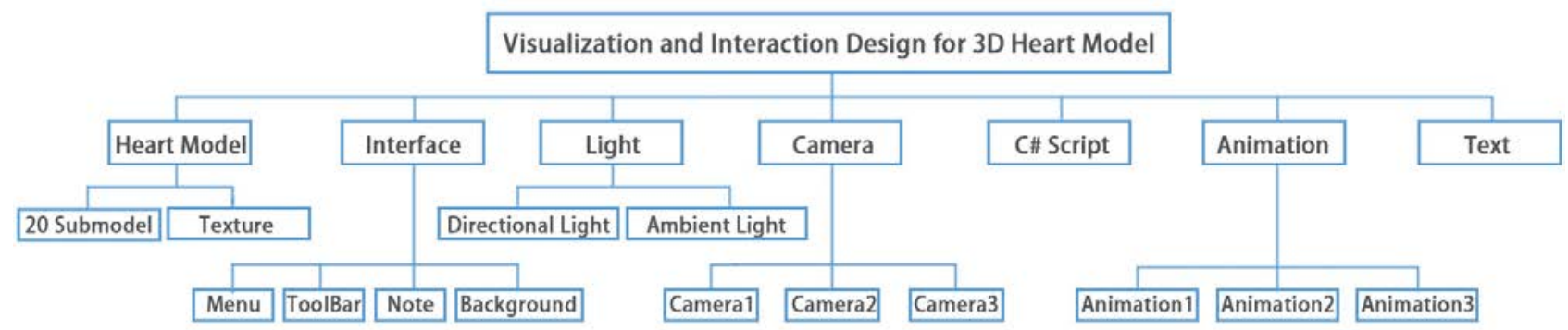

Fig. 2 Visualization and Interaction Design for 3D Heart Model

\section{Implementation of Interactive Technology}

Import all the materials, such as the design of pictures, animation videos and 3D heart models in Unity3D and integrate them. According to the architecture design, the layout of material in the window should be corrected, in addition, the interface and the heart models are configured with different cameras, and then adjust the depth value of each camera. The combined effect of the three cameras is as follows.

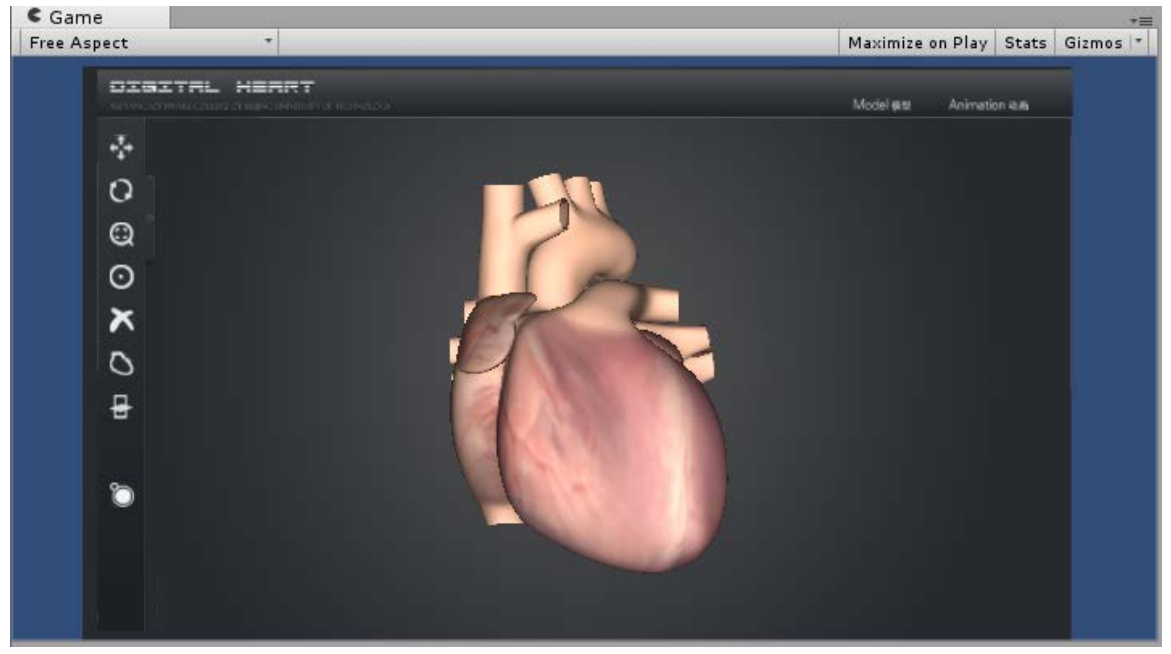

Fig. 3 Combined Effect of the Three Cameras 
In the menu bar, the interactive function of the "model" is mainly reflected in controlling the change of transparency, it highlights the model we choose by changing the transparent. In C\# script, we can define a class called AllTransparent (), change the property of "shader" to “Transparent/Diffuse”, and adjust the material color values to (1.0f, 1.0f, 1.0f, of), then it can realize the fully transparent effect automatically.

In the toolbar, there are seven kinds of interactive functions, respectively is translation, rotation, scaling, a separate display, delete, restore and the real time cross-section. We need to set static global variables and assignment for each state, such as GlobaiVar.curButtonState=0. After that, write C\# script corresponding to different state values. To realize the interaction of heart model, we need to add mesh colliders for all sub models, and then use ray detection method to control the sub model of which name is detected by the ray.

Some ray detection scripts are as follows.

if(isReceiveRay $==$ false)

\{

isReceiveRay=true;

_ray =Camera.main.ScreenPointToRay(Input.mousePosition);

if (Physics.Raycast(_ray, out_rayhit,_fDistance)) \{

\} print(_rayhit.collider.gameObject.name);

\}

\section{Result}

Through scripting, the interactive effects of menu bar includes "model" effect and "animation" effect, click "animation" can achieve the playback of animation videos, move the cursor to the "model”, a drop-down list will be shown, respectively, epicardium layer, cardiovascular layer, atrioventricular muscle layer and cardiac chambers layer. After clicking action, the "shader" attribute of the sub models which is not be selected will be changed to the value $30 \%$ according to the C\# script. The drop-down list effect and the clicked effect are as follows.

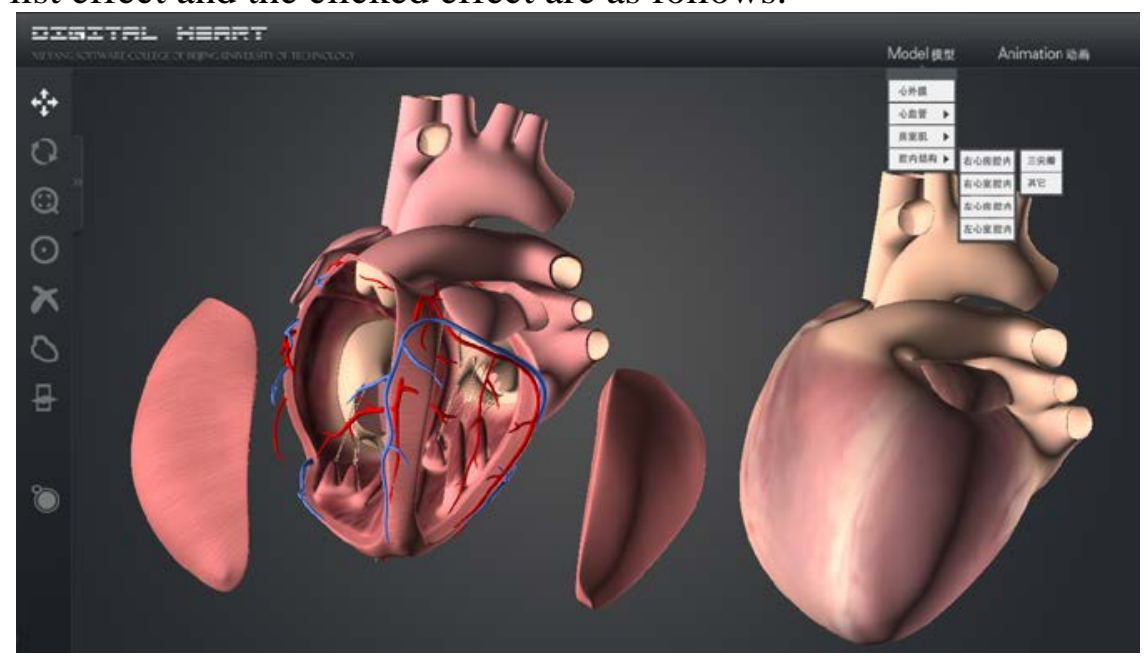

Fig. 4 Drop-down List Effect in the Menu Bar 


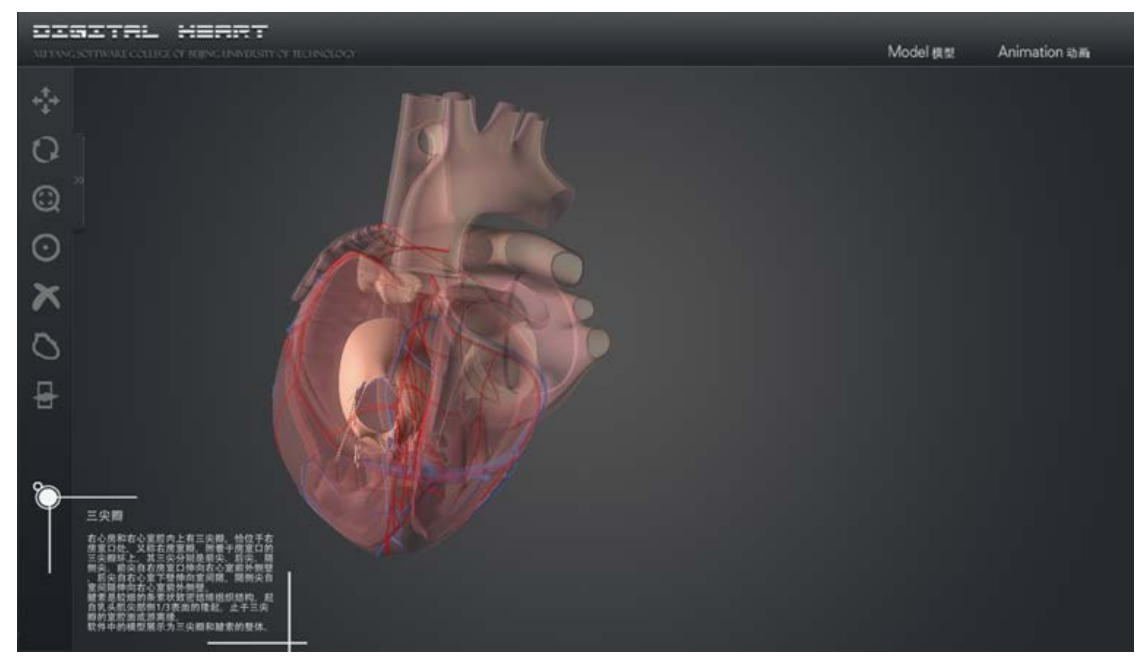

Fig. 5 Clicking Actions Effect in the Menu Bar

The interactive effects of toolbar are completed basically, when the seven tools icon is gray, we can achieve the functions such as translation, scaling and rotation for the whole models, when an icon is activated by clicking action, through the mouse operations, the sub model will be controlled according to the corresponding state value of "GlobaiVar.curButtonState" with the ray collision detection. Then we can implement a specific operation of a sub model, or to restore the initial models, to view the real-time profile of models. Some effects of toolbar are as shown below.

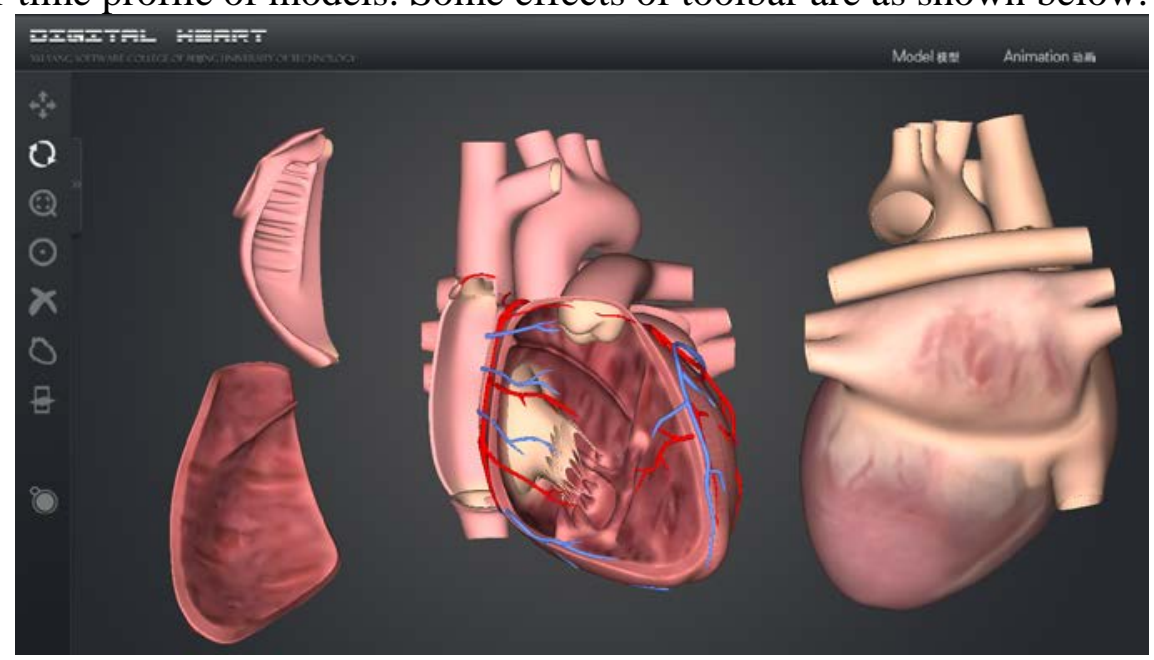

Fig. 6 Translation, Rotation Effect in the Toolbar

\section{Summary}

This research and the development program have achieved a kind of visualization and interaction of the digital 3D heart model, the research result is not only a static closed model, but an interactive and open system, it can render the atmosphere through the theme design, and allow users fall in it, give them more comprehensive understandings.

The research on visualization and interaction of digital 3D heart model is meaningful. First of all, the visualization of 3D heart model is very clear and closed to the real human heart. Second, it saves costs, does not waste precious human heart specimens, and doesn't have pollution. Again, visualization and interaction of digital 3D heart model has a great significance in the surgical treatment, its rich interactive means can help ordinary people especially medical researchers understanding the name, location, interrelationship, and dynamics knowledge of the heart internal structure, without question, it has a high practical value. 


\section{References}

[1] Lei Zhang, Yan Chi, Kuanquan Wang, Changqing Gai, Wangmeng Zuo, Yanjun Chen, Research on Interactive Visualization of 3D Cardiac Data. J. Intelligent Computer and Applications. 2013, 3(5):39-42.

[2] Peng Qiu, Jiao Li, Bing Liu, Hong Chen, Yan Pan, Ting Meng, Yuhua Zhang, Wenhong Sun, Lixin, Three-dimensional Reconstruction and Display of Heart Surface Model Based on Virtual Human Data. J. Biomedical Engineering Research. 2005,24(3):150-152.

[3] Mohammad Riduwana, Ahmad Hoirul Basori, Farhan Mohamed, Finger-based gestural interaction for exploration of 3D heart visualization . J . Procedia - Social and Behavioral Sciences. 2013, 97: $684-690$.

[4] Chunyan Zhong, Yanli Guo, Shaoxiang Zhang, Liwen Tan, Assessment on Three-dimensional Heart Models Based on Chinese Visible Vuman and Dual-source CT Data Base. J. China Medical Imaging Technology. 2011,27(10):2127-2130.

[5] Yafei Pang, Ming Yang, Research Status and Trend of Heart Modeling. J. Chinese Joumal of Medical Instrumentation. 2011,35(1):58-63.

[6] Information on http://www.3dscience.com

[7] Haijie Wang, Yuzhen Tan, Practical Heart Anatomy, first ed., Fudan University Press, China,2007. 